\title{
Padrões de Respostas Defensivas de Congelamento Associados a Diferentes \\ Transtornos DE ANSIEDADE ${ }^{1}$
}

\author{
Jesus Landeira-Fernandez ${ }^{2}$, Antonio Pedro de Mello $\mathrm{Cruz}^{3}$ e \\ Marcus Lira Brandão ${ }^{4}$ \\ Pontíficia Universidade Católica do Rio de Janeiro - PUC-Rio \\ Universidade Estácio de Sá - UNESA \\ Universidade de Brasília - UNB \\ Universidade de São Paulo - USP
}

Embora os transtornos de ansiedade sejam tipicamente humanos, eles apresentam correlações com determinadas reações de defesa de animais em situações de perigo. Este trabalho apresenta algumas relações entre determinados padrões da resposta defensiva de congelamento $e$ diferentes formas de transtornos de ansiedade. Em particular, destaca-se o isomorfismo entre a resposta de congelamento a estímulos contextuais associados a um estímulo aversivo e o transtorno de ansiedade generalizado. Evidências indicam também que a resposta de congelamento induzida pela estimulação elétrica da matéria cinzenta periaqueductal dorsal (MCPD) constitui um excelente modelo animal de ataque de pânico. A resposta de congelamento que surge imediatamente após estimulação da MCPD, capaz de produzir uma resposta de fuga, parece estar associada ao transtorno de pânico. Finalmente, é possível que a resposta de congelamento a estímulos previamente associados à estimulação elétrica da MCPD seja um modelo animal para o transtorno de pânico com agorafobia.

Descritores: Modelos animais de ansiedade. Resposta de congelamento.

Transtorno de ansiedade generalizada. Transtorno do pânico sem agorafobia. Transtorno do pânico com agorafobia.

1 Apoio financeiro do CNPq e FAPESP.

2 Docente do Departamento de Psicologia da PUC-Rio e da UNESA. Endereço eletrônico: landeira@psi.puc-rio.br

3 Docente do Instituto de Psicologia - UNB.

4 Docente da Faculdade de Filosofia, Ciências e Letras de Ribeirão Preto - USP. 


\section{J. Landeira-Fernandez, A. Pedro de Mello Cruz e M. Lira Brandão}

Dsicopatologias associadas aos transtornos de ansiedade estão entre os mais frequientes distúrbios da área da saúde mental. Estima-se que $20 \%$ da população mundial apresentem pelo menos um episódio relacionado a transtornos de ansiedade durante a vida (Almeida-Filho et al., 1997; Arikian \& Gorman, 2001). Levantamentos econômicos indicam que mais de $30 \%$ da verba destinada para a saúde mental nos Estados Unidos é exclusivamente utilizada para o tratamento de pacientes diagnosticados com algum tipo de transtorno de ansiedade (DuPont et al., 1996). Dessa forma, o estudo destes transtornos justifica-se não só pelo seu aspeto básico relacionado à descoberta de seus mecanismos patofisiológicos, como também por sua aplicabilidade clínica.

Os transtornos de ansiedade podem apresentar três grandes classes de sintomas: alterações na atividade consciente, agitação ou inibição comportamental e desregulação da fisiologia interna do corpo (homeostase). Dentre as alterações na consciência, a ansiedade pode provocar confusões ou distorções da percepção e modificar o significado de acontecimentos registrados por um indivíduo. Transtornos de ansiedade podem também interferir diretamente em processos de atenção e provocar prejuízos de aprendizagem e memória. Sensações de apreensão, hipervigilância, insônia e perda de concentração configuram ainda como outros sintomas associados à atividade mental consciente.

Sinais de inquietação, como andar de um lado para outro, movimentar as mãos, os pés e outras partes do corpo sem intenção aparente ilustram alguns dos sintomas comportamentais que acompanham a ansiedade. Expressões faciais características de apreensão, ou até mesmo redução ou inibição da atividade motora, podem ainda acompanhar alguns estados de ansiedade.

Além de toda esta sintomatologia cognitiva e comportamental, os estados de ansiedade são ainda capazes de promover alterações na regulação exercida pelo sistema simpático em determinados órgãos e glândulas, desencadeando sudorese emocional, palpitações, náuseas e sensações de vazio no estômago.

Apesar da plena expressão de reações de ansiedade ser um fenômeno tipicamente humano, é possível encontrar correlações entre estes transtornos de ansiedade e respostas de defesa que animais exibem frente a estímulos ou situações de perigo. Teorias que tentam relacionar reações de ansiedade em seres humanos com o comportamento de defesa em diferentes espécies animais tiveram origem com Darwin (1872/2000). Desde então, vários estudos têm 
definido a ansiedade como componente essencial de um sistema neural cuja origem se deu em mamíferos infra-humanos e que vem se mantendo ao longo da evolução das espécies. Esse sistema é responsável não apenas pela detecção de sinais de perigo no meio ambiente, mas também pela ativação de reações fisiológicas e comportamentais adequadas de defesa.

Com base nesta perspectiva, muitos modelos animais foram desenvolvidos com o objetivo de simular determinadas situações de perigo permitindo assim investigar experimentalmente aspectos etiológicos e eventuais formas de tratamento associados a determinados transtornos de ansiedade. Desde então, a utilização destes modelos tem sido decisiva para um melhor entendimento das bases neurais e farmacológicas envolvidas nas reações de defesa.

O presente artigo apresenta uma série de estudos que demonstram como determinados padrões da resposta de congelamento que animais exibem frente a situações de perigo podem estar relacionados a diferentes formas de transtornos de ansiedade. Antes de adentrarmos nos estudos experimentais que demonstram estas possíveis relações, é importante descrever como as diferentes patologias associadas à ansiedade podem ser classificadas clinicamente.

\section{A classificação da ansiedade e do pânico}

O estudo sistemático dos diferentes transtornos de ansiedade teve origem com Sigmund Freud (1856-1939). Através de observações clínicas, Freud (1886/1976) descreveu com clareza várias disfunções relacionadas com a ansiedade, dentre elas a crise aguda de angústia, a neurose de angústia e a expectativa ansiosa. Muito provavelmente, estas disfunções descritas por Freud no final do século XIX são conhecidas hoje, respectivamente, como ataque de pânico, transtorno de pânico e transtorno de ansiedade generalizada (TAG). Deve-se destacar, entretanto, que este sistema de classificação psicanalítico dos transtornos de ansiedade tornou-se, com o passar do tempo, altamente inferencial e especulativo, calcado quase que exclusivamente em pressupostos teóricos que ainda carecem de validação empírica.

Classificações mais objetivas dos transtornos de ansiedade podem ser encontradas no Manual Estatístico de Doenças Mentais DSM-IV-TR (Ame- 


\section{J. Landeira-Fernandez, A. Pedro de Mello Cruz e M. Lira Brandão}

rican Psychiatic Association, 2002), e na Classificação Internacional das Doenças Mentais (CID-10, Organização Mundial de Saúde [OMS], 1993). Estes manuais definem os diferentes transtornos de ansiedade através de critérios claros, possibilitando a formulação de diagnósticos mais precisos e confiáveis. De acordo com estes manuais, o TAG caracteriza-se pela presença de preocupação excessiva, persistente e desproporcional à realidade. Podem também ocorrer alta vigilância cognitiva, irritabilidade persistente e uma grande inquietação ou incapacidade de relaxar. Estados incontroláveis de preocupação e apreensão, além de uma variedade de sintomas somáticos em conseqüência de uma hiperatividade de sistemas fisiológicos como, por exemplo, diarréia crônica, dores musculares, disfunções gastrointestinais, dores de cabeça, palpitações ou taquicardias, são outros sintomas relacionados com o TAG.

Tanto o DSM-IV-TR quanto a CID-10 definem ataque de pânico como medo intenso ou terror, sentimento de morte iminente e expectativa de loucura, sensações acompanhadas de taquicardia, hiperventilação, asfixia, hipertensão arterial, dores no peito, tontura e náusea. $\mathrm{O}$ ataque tem início abrupto e duração curta, atingindo seu pico em torno de 10 a 20 minutos. Embora usualmente ocorra de forma espontânea e inesperada, não associada a qualquer situação específica, o ataque de pânico pode também ser precipitado por situações que o paciente julgar extremamente perigosas ou ameaçadoras.

O transtorno de pânico se caracteriza pela presença de ataques de pânico recorrentes e inesperados, seguidos por pelo menos um mês de preocupação persistente acerca de ter um outro ataque (American Psychiatric Association, 2002). Portanto, enquanto o ataque de pânico refere-se à crise aguda propriamente dita, momentaneamente acompanhada da sintomatologia acima descrita, o transtorno de pânico apresenta um componente adicional - a preocupação de novos ataques.

Graças aos estudos pioneiros do psiquiatra americano Donald Klein, sabe-se hoje que o transtorno de pânico e o TAG constituem-se em categorias nosológicas independentes. Em um destes estudos, Klein e Fink (1962) demonstraram que a imipramina, um antidepressivo tricíclico que atua inibindo e recaptação tanto da serotonina quanto da noradrenalina, atenuava significativamente a manifestação dos ataques de pânico, mas era ineficaz no TAG. Estudos que se seguiram não apenas confirmaram essas observações, como 
também demonstraram que os principais ansiolíticos benzodiazepínicos eram altamente eficazes no tratamento farmacológico do TAG, mas eram ineficazes ou, paradoxalmente, até mesmo precipitavam os ataques de pânico.

O transtorno de pânico pode ser seguido ou não de agorafobia, medo intenso de estar em locais públicos (do grego ágora, praça ou local público). Neste caso, o paciente evita sair de casa com medo de ter um novo ataque na ausência de alguém conhecido, recolhendo-se assim da vida social e profissional. Dessa forma, o DSM-IV-TR (American Psychiatic Association, 2002) considera a agorafobia como um transtorno secundário, classificado sempre em relação à história do pânico: transtorno de pânico sem agorafobia (simplesmente transtorno de pânico) e transtorno de pânico com agorafobia. Curiosamente, a relação destes transtornos na CID-10 (OMS, 1993) é completamente oposta, de maneira que a classificação considera a agorafobia como transtorno principal: agorafobia com a presença ou ausência de transtorno de pânico.

Estas quatro reações de ansiedade relativamente específicas - TAG, ataque de pânico e transtorno de pânico com ou sem agorafobia - podem encontrar grande semelhança com padrões diferenciados de expressão da resposta defensiva de congelamento em ratos. Em seguida apresentaremos algumas características desta resposta de defesa para que possamos apresentar algumas relações desta resposta com estes quatro transtornos de ansiedade.

\section{A reposta defensiva de congelamento}

A resposta de congelamento é relativamente simples e pode ser observada diretamente sem grandes dificuldades. Ela é definida como a ausência total de movimentos aparentes, exceto aqueles responsáveis pela respiração (Bolles \& Collier, 1976; Bouton \& Bolles, 1980). Vários estudos experimentais demonstraram que esta resposta é a reação de defesa mais comum quando ratos são expostos a situações de perigo (para uma revisão, ver Fanselow \& Gale, 2003).

Duas classes de estímulos podem ativar a resposta de congelamento: estímulos naturalmente ameaçadores, que são reconhecidos de maneira inata pelo animal, e estímulos de perigo condicionados, adquiridos através de pro- 


\section{J. Landeira-Fernandez, A. Pedro de Mello Cruz e M. Lira Brandão}

cessos de aprendizagem. O reconhecimento de um estímulo inato de perigo independe de qualquer processo prévio de aprendizagem e está presente em todos os indivíduos de uma mesma espécie. Por exemplo, embora não tenham qualquer tipo de experiência prévia com seus predadores naturais, ratos criados em laboratório não apenas reagem defensivamente a predadores do seu nicho ecológico, como por exemplo, um gato, (Lester \& Fanselow, 1985), como também a sinais ambientais da presença em potencial do predador, como o odor do gato (Fanselow, 1985), o cheiro deixado por um outro rato submetido a formas severas de estresse (Fanselow, 1985) ou a estimulação tátil de humanos na cabeça ou nas costas do animal (Fanselow \& Sigmundi, 1986). Em qualquer um desses casos, a resposta de congelamento pode ser observada quando ratos são expostos a esses estímulos pela primeira vez.

Além desses sinais de perigo, reconhecidos de forma inata pelo animal, outros estímulos também são capazes de eliciar a resposta de congelamento através de uma aprendizagem associativa. Por exemplo, um som, uma luz ou um contexto ambiental como um todo, podem adquirir a capacidade de induzir congelamento através de aprendizagem associativa com um estímulo aversivo incondicionado, como por exemplo, um choque elétrico. Este tipo de condicionamento é extremamente rápido, simples e duradouro. Num experimento típico que demonstra essa forma de aprendizagem, coloca-se um rato numa caixa experimental por alguns minutos e, em seguida, apresenta-se um choque elétrico de curta duração e pequena intensidade. No dia seguinte, recoloca-se o animal nessa caixa experimental sem que nenhum choque seja apresentado. O que se observa é que os estímulos contextuais que ficaram associados ao choque são capazes de ativar a resposta defensiva de congelamento. A intensidade da corrente elétrica utilizada no condicionamento contextual parece estar diretamente relacionada com a magnitude da resposta de congelamento (Landeira-Fernandez, 1996).

A resposta de congelamento produzida através do paradigma de condicionamento contextual aversivo está sob o controle dos estímulos contextuais previamente associados ao choque. Assim, no teste de mudança de contexto, que consiste na transferência do animal para uma outra caixa experimental após a apresentação do choque, observa-se que a resposta de congelamento está ausente (Fanselow, 1980). Outro procedimento que demonstra a natureza 
associativa desta resposta é o teste do choque imediato. Neste teste, o animal recebe um choque elétrico imediatamente após ser colocado pela primeira vez na caixa experimental. Neste caso, observa-se que animal apresenta as mesmas respostas incondicionadas ao choque, como pular, girar e vocalizar. Entretanto, a resposta de congelamento está totalmente ausente (Landeira-Fernandez, DeCola, Kim, \& Fanselow, 2006).

\section{O congelamento a estímulos contextuais aversivos como modelo do transtorno de ansiedade generalizada}

A modulação bidirecional da resposta de congelamento ao contexto como efeito de agentes farmacológicos que aumentam ou reduzem os sintomas de ansiedade em seres humanos tem sido utilizada para validar este procedimento como um modelo de TAG. Drogas altamente eficazes no tratamento farmacológico do TAG, como os ansiolíticos benzodiazepínicos, atenuam ou mesmo abolem a resposta de congelamento ao contexto em ratos em uma faixa de dose semelhante àquela utilizada clinicamente em humanos. Por outro lado, compostos ansiogênicos, como algumas beta-carbolinas, produzem sintomas de ansiedade em humanos e aumentam a magnitude da resposta condicionada de congelamento ao contexto em ratos (Conti, Maciver, Ferkany, \& Abreu, 1990). Estes resultados conferem um isomorfismo entre a resposta de congelamento ativada por estímulos contextuais associados a choques elétricos e o TAG.

A Tabela 1 apresenta as diferentes formas de congelamento e algumas de suas características comportamentais e neurofisiológicas bem como os possíveis transtornos de ansiedade associados a cada uma destas respostas. Conforme se pode observar na primeira linha da Tabela 1, a configuração desta resposta de congelamento induzida por processos de aprendizagem associativa não envolve piloereção ou exoftalmia, padrões geralmente observados na resposta de congelamento induzida de forma direta, sem a mediação de qualquer processo associativo, através da estimulação elétrica da matéria cinzenta periaquedutal dorsal (MCPD).

A primeira linha da Tabela 1 apresenta também o fato de que a resposta de congelamento condicionada está diretamente associada à matéria cinzen- 


\section{J. Landeira-Fernandez, A. Pedro de Mello Cruz e M. Lira Brandão}

ta periaquedutal ventral (MCPV), uma vez que a destruição desta área abole a expressão desta reposta (Fanselow et al., 1995). Projeções monosinápticas entre o complexo amigdalóide e a MCPV sugerem que a atividade neural responsável pela ativação da MCPV necessária para produzir a resposta de congelamento parte do complexo amigdalóide (Carrive, Lee, \& Su, 2000; Rizvi, Ennis, Behbehani, \& Shipley, 1991). De fato, diversos resultados indicam que a lesão de qualquer uma das áreas que compõem o complexo amigdalóide abole esta resposta de congelamento. Evidências recentes indicam que, enquanto a estimulação de neurônios gabaérgicos localizados no complexo amigdalóide reduz a ocorrência da resposta de congelamento a estímulos contextuais associados a choques elétricos, a estimulação de neurônios serotonérgicos nesta mesma estrutura promove um efeito oposto (Ruiz-Martinez, de Oliveira, \& Brandão, 2006).

Além de ativar a MCPV para produzir a resposta de congelamento, o complexo amigdalóide também envia projeções para neurônios hipotalâmicos de forma direta ou indireta através do núcleo intersticial lateral da estria terminal. Estas projeções são responsáveis pelo controle de reações hormonais que o animal emite frente a estímulos de perigo. Neurônios localizados no hipotálamo paraventricular secretam o hormônio liberador da corticotrofina que chega até a porção anterior da hipófise, glândula situada na base do cérebro. A hipófise, por sua vez, libera na corrente sanguiínea o hormônio adrenocorticotrófico (adrenal corticotrophic hormone; ACTH) que chega até a porção cortical da glândula supra-renal. Ali, o ACTH promove a liberação no sangue de glicocorticoides e outros hormônios esteróides. Esses agentes químicos produzem uma série de alterações fisiológicas preparando o organismo para possíveis danos que os estímulos de perigo possam causar ao sujeito. Conforme ilustrado na primeira coluna da Tabela 1, esta reação do eixo hipotalâmico-hipofisário-adrenal frente a estímulos contextuais aversivos também é uma característica importante observada no TAG.

Finalmente, deve-se indicar que a resposta de congelamento que um animal apresenta a estímulos contextuais aversivos constitui uma postura tensa. Esta idéia pode ser testada experimentalmente através de um procedimento capaz de produzir a potencialização do reflexo do sobressalto a um estímulo sonoro quando o animal se encontra numa situação de ameaça. Brown, Kalish e Faber (1951) observaram que a amplitude do reflexo de sobressalto a 


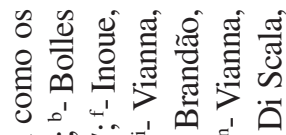

ஜ

응 ๙

ปै

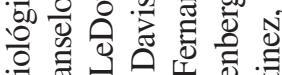

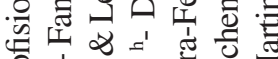

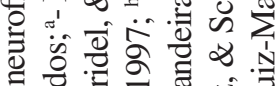

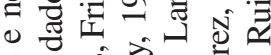

.

䒕

छ

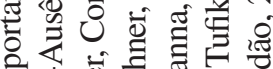

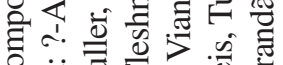

ठํं

चै च खें

次

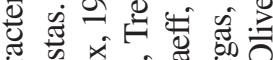

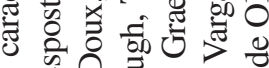

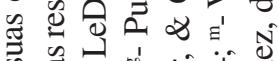

क चै ఐ क

ช ชิ

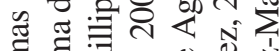

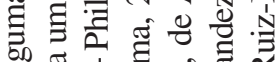

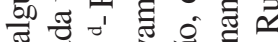

- ฮั่

을

ฮี

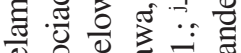

D

ठี तี

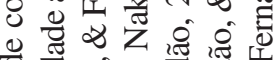

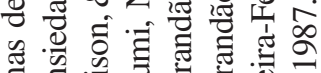

है तี

유 छే

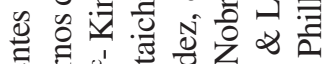

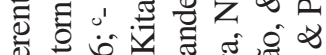

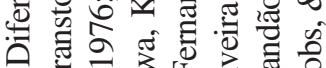

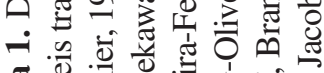

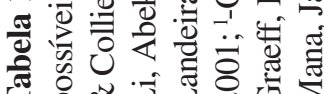

\begin{tabular}{|c|c|c|c|c|c|}
\hline \multicolumn{2}{|c|}{ 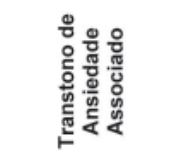 } & 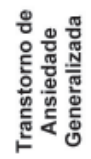 & 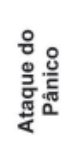 & 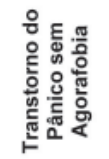 & 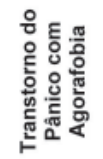 \\
\hline \multirow{8}{*}{ 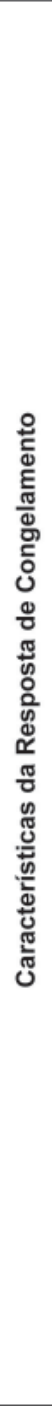 } & 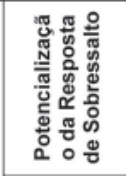 & है & $\sim$ & $\sim$ & $\sim$ \\
\hline & 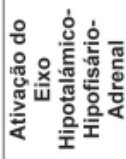 & $\stackrel{\text { है }}{\omega}$ & $\begin{array}{l}E_{0} \\
\frac{2 \pi}{Z}\end{array}$ & ح. & $\sim$ \\
\hline & 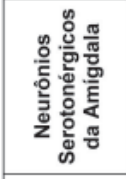 & 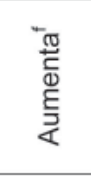 & 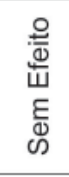 & 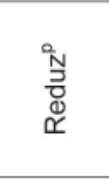 & $\sim$ \\
\hline & 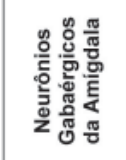 & $\begin{array}{l}\stackrel{\oplus}{J} \\
\stackrel{D}{0} \\
\check{1}\end{array}$ & 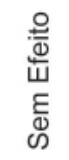 & $\begin{array}{l}\stackrel{ }{N} \\
\stackrel{0}{0} \\
\check{\Upsilon}\end{array}$ & c. \\
\hline & 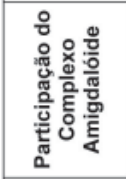 & 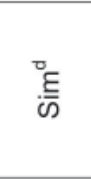 & $\begin{array}{l}\overline{0} \\
\stackrel{2 \pi}{Z}\end{array}$ & $\stackrel{\circ}{\circ}$ & c. \\
\hline & 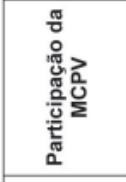 & $\stackrel{\circ}{\circ}$ & $\begin{array}{l}x_{0} \\
\text { 更 } \\
Z\end{array}$ & ค. & $\sim$ \\
\hline & 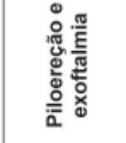 & $\begin{array}{l}\stackrel{0}{0} \\
\text { III } \\
Z\end{array}$ & $\bar{\xi}$ & $\sim$ & $\sim$ \\
\hline & 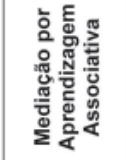 & $\stackrel{\text { "E }}{\stackrel{E}{\omega}}$ & io & $\begin{array}{l}\text { [o } \\
\text { in } \\
Z\end{array}$ & $\stackrel{\text { E. }}{\omega}$ \\
\hline & 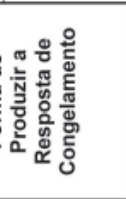 & 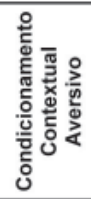 & 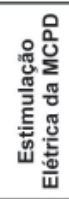 & 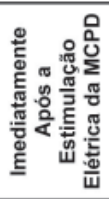 & 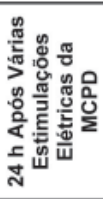 \\
\hline
\end{tabular}


um estímulo auditivo podia ser acentuada na presença de um estímulo previamente associado a um choque elétrico. Hoje existe uma série de evidências mostrando uma alta correlação entre a magnitude da potencialização do sobressalto e a resposta de congelamento a estes estímulos contextuais aversivos (Leaton \& Borszcz, 1985). Este conjunto de resultados indica que animais, ao serem expostos a estímulos contextuais aversivos, apresentam uma resposta de congelamento (inibição comportamental), mas quando um estímulo sonoro é apresentado nessa situação, ocorre uma exacerbação do reflexo de sobressalto (ativação comportamental).

\section{O congelamento à estimulação da MCPD como modelo do ataque de pânico}

A resposta defensiva de congelamento pode ainda ser diretamente provocada pela estimulação de determinadas áreas cerebrais. Por exemplo, a estimulação da MCPD de forma gradual produz, inicialmente, uma resposta de congelamento. A partir de uma determinada intensidade, a resposta de congelamento desaparece tendo início uma resposta de fuga muito intensa (Brandão, de Aguiar, \& Graeff, 1982).

Várias evidências apontam para o fato de que a resposta de congelamento induzida pela estimulação elétrica da MCPD é um excelente modelo animal de ataque de pânico. Agentes químicos capazes de reduzir a ocorrência de ataques de pânico em seres humanos, tais como, a clomipramina e a fluoxetina, aumentam a intensidade da estimulação elétrica da MCPD para produzir as respostas de congelamento em ratos. Por outro lado, drogas que aumentam a incidência de ataques de pânico em seres humanos, como por exemplo, o pentilenotetrazol, reduzem a intensidade da corrente elétrica na MCPD necessária para induzir a resposta de congelamento (Schenberg, Bittencourt, Sudre, \& Vargas, 2001; Jenck, Moreau, \& Martin, 1995). De fato, a estimulação elétrica da MCPD em seres humanos é capaz de produzir sintomas muito semelhantes aos ataques de pânico como, por exemplo, sentimentos de terror e morte iminente acompanhado pelo aumento de batimentos cardíacos, dor difusa na face e no peito (Nashold, Wilson, \& Slaughter, 1969).

Conforme se pode observar na segunda coluna da Tabela 1, a resposta de congelamento induzida pela estimulação elétrica da MCPD também produz 
piloereção e exoftalmia. Esta é uma característica específica desta resposta de congelamento e difere daquela induzida por estímulos contextuais associados a choques elétricos.

Estudos do nosso grupo indicaram que a resposta de congelamento induzida estimulação elétrica da MCPD independe da integridade da MCPV, uma vez que lesões nesta estrutura capazes de reduzir a resposta de congelamento a estímulos contextuais associados com choque elétricos não alteraram a ocorrência desta resposta produzida pela estimulação da MCPD (Vianna, Graeff, Landeira-Fernandez, \& Brandão, 2001). Da mesma forma, a lesão do complexo amigdalóide capaz de abolir a resposta de congelamento a estímulos contextuais aversivos não produziu qualquer impacto nesta resposta induzida pela estimulação da MCPD (Oliveira, Nobre, Brandão, \& Landeira-Fernandez, 2004). Tais resultados encontram-se na segunda linha da Tabela 1 e indicam que a resposta de congelamento associada à estimulação da MCPD não depende da ativação de estruturas neurais intimamente relacionadas com a expressão da ansiedade antecipatória caracterizada pelo condicionamento contextual aversivo.

A segunda linha da Tabela 1 apresenta ainda o fato de que a estimulação da MCPD não ativa o eixo hipotalâmico-hipófisário-adrenal (Vargas et al., 2001). Estes dados são importantes, uma vez que ataques de pânicos induzidos em seres humanos através do lactato de sódio ou do dióxido de carbono também não são capazes de ativar este eixo cerebral responsável pelo controle do sistema hormonal (para uma revisão, ver Graeff, Garcia-Leal, Del-Ben, \& Guimarães, 2005). Este conjunto de resultados fortalece a sugestão de que a resposta de congelamento induzida pela estimulação da MCPD constitui, de fato, um modelo animal seletivo de ataque de pânico, mas não de TAG.

\section{O congelamento pós-estimulação da MCPD como modelo do transtorno de pânico}

A estimulação elétrica da MCPD, além de produzir uma resposta de congelamento, pode também levar a uma reposta de fuga graças ao aumento da intensidade desta estimulação elétrica. Entretanto, esta resposta de fuga desaparece assim que a estimulação elétrica da MCPD cessa, tendo início uma nova a resposta de congelamento. Sabe-se muito pouco acerca desta resposta 


\section{J. Landeira-Fernandez, A. Pedro de Mello Cruz e M. Lira Brandão}

inibitória que ocorre após a estimulação elétrica da MCPD. Entretanto, sabe-se que ela se diferencia bastante daquela induzida diretamente pela estimulação da MCPD, bem como daquela induzida por estímulos contextuais aversivos. Conforme se pode observar na terceira linha da Tabela 1 , a resposta de congelamento que tem início após a estimulação da MCPD independe de qualquer processo associativo, diferenciando-se, assim, daquela induzida por estímulos contextuais aversivos (Vianna, Landeira-Fernandez, \& Brandão, 2001). Mais importante ainda, esta resposta de defesa está sob influência do complexo amigdalóide, ao contrário daquela induzida diretamente através da estimulação da MCPD. Microinjeções de um agonista gabaérgico na amígdala reduziram esta resposta de congelamento de forma similar à resposta de congelamento induzida por estímulos contextuais aversivos (Ruiz-Martinez et al., 2006).

Calcado no fato de que a resposta de congelamento que tem início após a estimulação da MCPD se diferencia daquela induzida diretamente pela estimulação da MCPD (modelo animal do ataque de pânico), bem como daquela induzida por estímulos contextuais aversivos (modelo animal de TAG), é possível levantar a hipótese de que esta resposta possa representar um modelo animal de outro transtorno de ansiedade. Observações clínicas apontam para diferenças importantes entre o ataque e o transtorno de pânico, especialmente no que diz respeito a uma reação intensa de ansiedade (ataque de pânico) e a apreensão e preocupação constante que pacientes com transtorno de pânico apresentam em sofrerem um novo ataque de pânico. Esta preocupação constante caracteriza um importante componente de ansiedade antecipatória presente no transtorno de pânico, mas ausente no ataque de pânico.

O fato de o complexo amigdalóide ser uma estrutura neural relacionada com ansiedade antecipatória e exercer uma modulação sobre a resposta de congelamento observada após a estimulação da MCPD, mas não aquela induzida pela estimulação direta desta estrutura, sugere que esta resposta pode estar associada ao transtorno, mas não ao ataque de pânico. Resultados farmacológicos tendem também a corroborar essa hipótese. Por exemplo, agonistas serotonérgicos utilizados no tratamento do pânico paradoxalmente aumentam os sintomas de ansiedade. De forma análoga, a microinjeção de um agonista serotonérgico no complexo amigdalóide foi capaz de reduzir a expressão da resposta de congelamento após a estimulação da MCPD, mas aumentou 
a expressão do congelamento a estímulos contextuais associados a choques elétricos (Ruiz-Martinez, 2006).

\section{Congelamento a estímulos contextuais associados a estimulações da MCPD como modelo do transtorno de pânico com agorafobia}

Embora a resposta de congelamento que tem início após a estimulação elétrica da MCPD não dependa de qualquer processo de aprendizagem associativa, é possível utilizar a estimulação elétrica desta área como um estímulo incondicionado com objetivo de produzir um condicionamento contextual aversivo. Neste caso, são necessárias várias estimulações elétricas da MCPD para que este tipo de aprendizagem associativa possa ocorrer. Por exemplo, Castilho e Brandão (2001) demonstraram que estímulos contextuais associados à estimulação elétrica da MCPD foram capazes de produzir respostas de congelamento quando apresentados sozinhos um dia depois.

Como se pode observar na quarta linha da Tabela 1, ainda não existem informações conclusivas sobre a natureza desta resposta defensiva de congelamento, a não ser o fato de ela estar sob o controle de algum tipo de aprendizagem associativa. Entretanto, deve-se destacar que esta forma de aprendizagem associativa ocorre de forma relativamente lenta e alguns animais sequer a desenvolvem (Castilho \& Brandão, 2001). Curiosamente, este é exatamente o mesmo padrão observado em pacientes com transtornos do pânico. Enquanto alguns desenvolvem formas severas de ansiedade, chegando mesmo a se sentirem impossibilitados de saírem de casa, caracterizando assim o transtorno de pânico com agorafobia, em outros a aprendizagem associativa é tão fraca que estes pacientes com transtorno de pânico jamais desenvolvem qualquer tipo de agorafobia. Dessa forma, pode-se especular que a resposta de congelamento associada a estímulos contextuais previamente associados a estimulações elétricas da MCPD pode servir de modelo animal para o transtorno de pânico com agorafobia. Está é uma idéia nova que pode ser facilmente testada experimentalmente. Na verdade, a quantidade de pontos de interrogação presentes na Tabela 1 expressa a real dimensão daquilo que ainda necessita ser pesquisado para diferenciar estas respostas de congelamento e eventualmente utilizálas como modelos específicos de ansiedade. 
Landeira-Fermandez, J., Cruz, A. P. M., \& Brandão, M. L. (2006). Distinct patterns of freezing behavior associated with different anxiety disorders. Psicologia USP, 17(4), 175-192.

\begin{abstract}
Although anxiety disorders are exclusive to humans, it is possible to find correlation between these disorders and defensive responses that animals present when facing dangerous situations. The present article presents a series of evidence that indicate that different freezing defensive patterns might be associated with specific anxiety disorders. Particularly, there is an excellent isomorphism between freeing response to contextual stimuli associated with electrical shocks and generalized anxiety disorder. Much evidence also shows that freezing response triggered directly through the electrical stimulation of the dorsal periaqueductal gray matter $(\mathrm{dPAG})$ is an excellent animal model of panic attack. Moreover, freezing response observed after the interruption of the electrical stimulation of the dPAG which triggers an escape response seems to be associated with panic disorder. Finally, it is hypothesized that freezing to contextual stimuli previously associated with intense electrical stimulation of the dPAG might be related to panic disorder with agoraphobia.
\end{abstract}

Index terms: Animal models of anxiety. Freezing response. Generalized anxiety disorder. Panic disorder without agoraphobia. Panic disorder with agoraphobia.

Landeira-Fermandez, J., Cruz, A. P. M., \& Brandão, M. L. (2006). Des patterns de comportement d'immobilisation distincts sont associés avec différents troubles anxieux. Psicologia USP, 17(4), 175-192.

Résumé: Bien que les troubles anxieux soient propres aux humains, il est possible d'établir un parallèle entre ces troubles et les réponses défensives que les animaux présentent lorsqu'ils sont confrontés à des situations dangereuses. Dans cet article, une série d'arguments est présentée indiquant que différents patterns d'immobilisation défensive sont associés avec des troubles anxieux spécifiques. En particulier, il y a un excellent isomorphisme entre la réponse d'immobilisation aux stimuli contextuels aversives et le trouble d'anxiété généralisée. De plus, le comportement d'immobilisation déclenché directement par la stimulation de la substance grise périaqueducale dorsale (dPAG) est un excellent modèle animal 


\section{Congelamento e Transtornos de Ansiedade}

d'attaque de panique. D'autre part, la réponse d'immobilisation observée après interruption de la stimulation électrique du dPAG semble associée avec le trouble panique. Finalement, l'hypothèse est proposée que l'immobilisation déclenchée par les stimuli contextuels précédemment associés avec la stimulation électrique du dPAG pourrait être mise en parallèle avec le trouble panique avec agoraphobie.

Mots clés: Modèles animaux d'anxiété. Réponse d'immobilisation.

Trouble d'anxiété généralisée. Trouble panique sans

agoraphobie. Trouble panique avec agoraphobie.

\section{Referências}

Almeida-Filho, N., Mari, J. J., Coutinho, E., França, J. F., Fernandes, F., Andreoli, S. B. et al. (1997). Brazilian multicentric study os psychiatric morbity. Bristish Journal of Psychiatry, 171, 524-529.

American Psychiatric Association. (2002). DSM-IV-TR: manual estatístico de transtornos mentais. Porto Alegre: Artmed.

Arikian, S. R., \& Gorman, J. M. (2001). A review of the diagnosis, pharmacologic treatment, and economic aspects of anxiety disorders. Prim care companion. Journal of Clinical Psychiatry, 3, 110-117.

Bolles, R. C., \& Collier, A. C. (1976). The effect of predictive cues of freezing in rats. Animal Learning and Behavior, 4, 6-8.

Bouton, M. E., \& Bolles, R. C. (1980). Conditioned fear assessed by freezing and by the suppression of three different baselines. Animal Learning and Behavior, 8, 429434.

Brandão, M. L., de Aguiar, J. C., \& Graeff, F. G. (1982). GABA mediation of the anti-aversive action of minor tranquilizers. Pharmacology Biochemestry and Behavior, 16, 397-402.

Brown, J. S., Kalish, H. J., \& Faber, J. E. (1951). Conditioned fear as revealed by magnitude of startle response to an auditory stimulus. Journal of Experimental Psychology, 41, 317-328.

Carrive, P., Lee, J., \& Su, A. (2000). Lidocaine blockade of amygdala output in fearconditioned rats reduces Fos expression in the ventrolateral periaqueductal gray. Neuroscience, 95, 1071-1080.

Castilho, V. M., \& Brandão, M. L. (2001). Conditioned antinociception and freezing using electrical stimulation of the dorsal periaqueductal gray or inferior colliculus as unconditioned stimulus are differentially regulated by 5-HT2A receptors in rats. Psychopharmacology (Berl), 155, 154-162. 


\section{J. Landeira-Fernandez, A. Pedro de Mello Cruz e M. Lira Brandão}

Conti, L. H., Maciver, C. R., Ferkany, J. W., \& Abreu, M. E. (1990). Footshock-induced freezing behavior in rats as a model for assessing anxiolytics. Psychopharmacology (Berl), 102, 492-497.

Darwin, C. (2000). A expressão das emoções nos homens e nos animais. São Paulo: Companhia das Letras. (Trabalho original publicado em 1872)

Davis, M. (1989). Sensitization of the acoustic startle reflex by footshoc. Behavioral Neuroscience, 103, 495-503.

Di Scala, G., Mana, M. J., Jacobs, W. J., \& Phillips, A. G. (1987). Evidence of Pavlovian conditioned fear following electrical stimulation of the periaqueductal grey in the rat. Physiology and Behavior, 40, 55-63.

DuPont, R. L., Rice, D. P., Miller, L. S., Shiraki, S. S., Rowland, C. R., \& Harwood, H. J. (1996). Economic costs of anxiety disorders. Anxiety, 2, 167-172.

Fanselow, M. S. (1980). Conditional and unconditional components of post-shock freezing. Pavlovian Journal of Biological Science, 15, 177-182.

Fanselow, M. S. (1985). Odors released by stresses rats produced opioid analgesia in unstressed rats. Behavioral Neuroscience, 99, 589-592.

Fanselow, M. S., DeCola, J. P., DeOca, B., \& Landeira-Fernandez, J. (1995). Ventral and dorsolateral regions of the midbrain periaqueductal gray control different stages of defensive behavior: Dorsolateral PAG lesions enhance the defensive freezing produced by massed and immediate shock. Aggressive Behavior, 21, 63-77.

Fanselow, M. S., \& Gale, G. D. (2003). The amygdala, fear, and memory. Annals of the New York Academy of Science, 985, 125-134.

Fanselow, M. S., \& Sigmundi, R. A. (1986). Species specific danger signals, endogenous opioid analgesia, and defensive behavior. Journal of Experimental Psychology: Animal Behavior Processes, 12, 301-309.

Freud, S. (1976). Sobre os critérios para destacar da neurastenia uma síndrome particular intitulada neurose de angústia Neurastenia e neurose de Angustia. In S. Freud, Edição standard brasileira das obras psicológicas completas de Sigmund Freud (Vol. 3, pp. 107-136). Rio de Janeiro: Imago. (Trabalho original publicado em 1895)

Graeff, F. G., Garcia-Leal, C., Del-Bem, C. M., \& Guimaraes F. S. (2005). Does the panic attack activate the hypothalamic-pituitary-adrenal axis? Anais da Academia Brasileira de Ciências, 77, 477-91.

Inoue, T., Li, X. B., Abekawa, T., Kitaichi, Y., Izumi, T., Nakagawa, S. et al. (2004). Selective serotonin reuptake inhibitor reduces conditioned fear through its effect in the amygdala. European Journal of Pharmacology, 497, 311-316. 


\section{Congelamento e Transtornos de Ansiedade}

Jenck F, Moreau, J. L., \& Martin, J. R. (1995). Dorsal periaqueductal gray-induced aversion as a simulation of panic anxiety: Elements of face and predictive validity. Psychiatry Research, 57, 181-191.

Kim, J. J., Rison, R. A., \& Fanselow, M. S. (1993). Effects of amygdala, hippocampus, and periaqueductal gray lesions on short- and long-term contextual fear. Behavioral Neuroscience, 107, 1093-1098.

Klein, D. F., \& Fink, M. (1962). Psychiatric reaction patterns to imipramine. American Journal of Psychiatry, 119, 432-438

Landeira-Fernandez, J. (1996). Pavlovian context conditioning. Brazilian Journal of Medical and Biological Research, 29, 149-173.

Landeira-Fernandez, J., DeCola, J. P., Kim, J. J., \& Fanselow, M. S. (2006). Immediate shock deficit in fear conditioning: Effects of shock manipulations. Behavioral Neuroscience, 120, 873-879.

Leaton, R. N., \& Borszcz, G. S. (1985). Potentiated startle: Its relations to freezing and shock intensity in rats. Journal of Experimental Psychology: Animal Behavioral Processes, 11, 421-428.

Leste, L., \& Fanselow, M. S. (1985). Exposure to a cat produces opioid analgesia in rats. Behavioral Neuroscience, 99, 756-759.

Muller, J., Corodimas, K. P., Fridel, Z., \& LeDoux, J. E. (1997). Functional inactivation of the lateral and basal nuclei of the amygdala by muscimol infusion prevents fear conditioning to an explicit conditioned stimulus and to contextual stimuli. Behavioral Neuroscience, $111,683-691$.

Nashold, B. S. Jr., Wilson, W. P., \& Slaughter, D. G. (1969). Sensations evoked by stimulation in the midbrain of man. Journal of Neurosurgery, 30, 14-24.

Oliveira, L. C., Nobre, M. J., Brandão, M. L., \& Landeira-Fernandez, J. (2004). Role of amygdala in conditioned and unconditioned fear generated in the periaqueductal gray. Neuroreport, 15, 2281-2285.

Organização Mundial de Saúde. (1993). Classificação de transtornos mentais e de comportamento da CID-10. Descrições clínicas e diretrizes diagnósticas. Porto Alegre: Artes Médicas.

Phillips, R. G., \& LeDoux, J. E. (1990). Differential contribution of amygdala and hippocampus to cued and contextual fear conditioning. Behavioral Neuroscience, 106, 274-285.

Pugh, C. R., Tremblay, D., Fleshner, M., \& Rudy, J. W. (1997). A selective role for corticosterone in contextual-fear conditioning. Behavioral Neuroscience, 111, 503-511. 
Rizvi, T. A., Ennis, M., Behbehani, M., \& Shipley, M., T. (1991). Connections between the central nucleus of the amygdala and the midbrain periaqueductal gray: Topography and reciprocity. Journal of Comparative Neurology, 303, 121-131.

Ruiz-Martinez, R. C. (2006). Regulação serotoninérgica e gabaérgica nos núcleos amigdalóides no medo condicionado e incondicionado. Tese de Doutorado, Programa de Psicobiologia, Universidade de São Paulo, Ribeirão Preto, SP.

Ruiz-Martinez, R. C., de Oliveira, A. R., \& Brandão, M. L. (2006). Conditioned and unconditioned fear organized in the periaqueductal gray are differentially sensitive to injections of muscimol into amygdaloid nuclei. Neurobiology of Learning and Memory, $85,58-65$.

Schenberg, L. C., Bittencourt, A. S., Sudre, E. C., \& Vargas, L. C. (2001). Modeling panic attacks. Neuroscience Biobehavioral Review, 25, 647-659.

Vargas, L. C., Silva, S. R., Reis, A. M., Tufik, S., Carobrez, A. P., \& Schenberg, L. C. (2001). Pharmacological and endocrinological properties of intracranially-induced panic-like behaviors. Society fo Neuroscience Abstract, 27, 858.11.

Vianna, D. M. L., Graeff, F. G., Brandão, M. L., \& Landeira-Fernandez, J. (2001) Defensive freezing evoked by electrical stimulation of the periaqueductal gray: Comparison between dosolateral and ventrolateral regions, NeuroReport, 18, 4109-4112.

Vianna, D. M. L., Graeff, F. G., Landeira-Fernandez, J., \& Brandão, M. L. (2001). Lesion of the ventral periaqueductal gray reduces conditioned fear but does not change freezing induced by stimulation of the dorsal periaqueductal gray. Learning \& Memory, 8, 164-169.

Vianna, D. M., Landeira-Fernandez. J., \& Brandão, M. L. (2001). Dorsolateral and ventral regions of the periaqueductal gray matter are involved in distinct types of fear. Neuroscience Biobehavioral Review, 25, 711-719.

Recebido em: 20/11/2006

Aceito em: 02/12/2006 